\title{
Assessment of automatic generation methods of conformance test suites in an industrial context
}

Laurent Doldi, Vincent Encontre

VERILOG, 150 rue Nicolas Vauquelin, BP 1310, F-31106 Toulouse Cedex, France

(doldi,encontre)@verilog.fr

Jean-Claude Fernandez

INRIA, Vérimag, Miniparc Zirst, rue Lavoisier, F-38330 Montbonnot Saint-Martin, France

Jean-Claude.Fernandez@imag.fr

Thierry Jéron

INRIA, IRISA/Pampa, Campus de Beaulieu, F-35042 Rennes, France

Thierry.Jeron@irisa.fr

Sylvie Le Bricquir, Nathalie Texier

CAP SESA REGIONS, rue de la Rigourdière, F-35517 Cesson-Sévigné Cedex, France

(slebricq,ntexier)@ecom.cgs.fr

Marc Phalippou

FRANCE TELECOM / CNET, Technopole Anticipa, 2 avenue Pierre Marzin, F-22307

Lannion Cedex, France, phalippo@LANNION.cnet.fr

\begin{abstract}
In this paper we describe three experiments in the automatic generation of conformance abstract test suites for protocol testing. The methods and tools have been applied to a same industrial protocol, the DREX protocol. This protocol has been specified in SDL language. Abstract conformance test suites have been generated in TTCN language. The study proved that the technology transfer to obtain a commercial tool is now possible.
\end{abstract}

\section{Keywords}

SDL (Specification and Description Language), ISO 9646 standard, IUT (Implementation Under Test), Test purposes, Abstract test suites, TTCN (Tree and Tabular Combined Notation), MSC (Message Sequence Charts). 


\section{INTRODUCTION}

Conformance testing is a major issue of strategic importance for the acceptance of OSI and other products based on standards for open systems. The central point in a conformance testing process lies in the availability of a test suite which must be closely related to the protocol (or system) specification. In spite of an important research effort in this area, this problem has not yet received a satisfactory solution. Many methods have been published, but there is no available commercial tool today able to process industrial SDL specifications in order to compute test suites in a really automated way.

This paper presents the conclusions of an experimental study whose objective was to evaluate and compare several available test generation techniques. Three prototype tools have been applied to the SDL specification of a real industrial protocol, and their outputs have been analysed and compared with manually written test suites. This experiment shows that by taking the best part of state-of-the-art research on test generation, it is possible to define now the architecture of an operational test generation tool. Our contribution includes in particular the following key points:

1. produce useful tests: many test generation methods are criticized by practitionners because they define non usable tests (too many, not checking the right parts,...). To avoid this trouble, we started from an existing industrial protocol, and we compared the results of our experiment with previously manually generated test suites. This guarantees that our results are really close to the users' needs.

2. use efficient algorithms: many test generation methods are unable to process industrial specifications for performance reasons (state explosion, computation time,...). We investigated how to reuse powerful algorithms and techniques (in particular on-the-fly computations) from protocol verification domain. In this way we beneficiate from many years of experience in this area, and we avoid reinventing the wheel.

3. combine complementary methods: we discovered advantages and drawbacks in each one of the three test generation methods which we have studied. The way we propose to combine them for building an "ideal" tool (see section 4) takes a best profit from all these combined ideas.

4. produce qualitative and quantitative gains: the use of formal methods increases the confidence in the automatically generated test cases. The automatic methods require time to specify the protocol and to formalize test purposes. But this is amply balanced by the benefit in time of automatic methods.

This paper is organized as follows: section 2 summarizes the context and the objectives of this study. Section 3 gives some details about the three tools which have been used in our experiment. Section 4 presents the results of the experiment. Finally section 5 evaluates what could be expected from a combination of the best part of each tool and analyses the perspectives for an industrial transfer of the results in order to obtain a commercial tool.

\section{CONTEXT AND OBJECTIVES}

\subsection{Context and objectives of the study}

The experiments related in this paper have been conducted during an industrial contract for the Délégation Générale pour l'Armement (DGA) of the French Ministry of Defence. Partners of this contract are VERILOG, CNET (Centre National d'Etudes des Télécommunications), CAP SESA, and two research groups of INRIA (Institut National de Recherche en Informatique et Automatique). This study started in November 1994 and ended in November 1995. 
The goal of the study is to prove that the automatic generation of conformance test sequences from a formal specification of a protocol and test purposes is feasible and profitearning in industrial context.

\subsection{Methods and tools}

Three methods and tools have been studied and/or developed : TGV, TOPIC V2 and TVEDA V3. TOPIC V2 is a prototype of VERILOG based on a previous prototype TOPIC (Montiel, 1994). TGV (for Test Generation from Verification techniques) from INRIA is based on verification techniques such as synchronous product and on-the-fly verification. TGV did not exist at the beginning of the study. TVEDA V3 from CNET (Phalippou, 1994) incorporates two distinct generation techniques; the technique used during this study is based on syntactic transformation of the specification.

From the methodological point of view, the experiment is in keeping with the general pattern of abstract tests suites specification as described in ISO 9646 standard and take also into account the processes used by practitioners. We use a formal description of the protocol, given in the SDL language (CCITT, 1988). We also use test purposes to select test cases. A test case is a set of sequences of interactions related to a particular test purpose.

In order to compare the methods and tools, these three tools had to generate abstract test suites, starting with the same SDL specification and test purposes in natural language for the DREX protocol described below.

\subsection{The DREX protocol and its specification}

The protocol used for our expriments is called the DREX protocol. This protocol has been chosen for three principal reasons:

- the protocol had to be an industrial one in order to prove feasibility of automatic test generation methods on realistic specifications,

- an SDL specification of a similar protocol was already available, written by CAP SESA,

- hand written test suites had already been produced, also by CAP SESA and this would serve as a basis for comparison with automatically generated test suites.

The DREX protocol is a military protocol which alllows the access to the transit network of the French Army defined in the framework of Integrated Services Military Networks. It is the analogous of the D protocol in the civil framework, with military features.

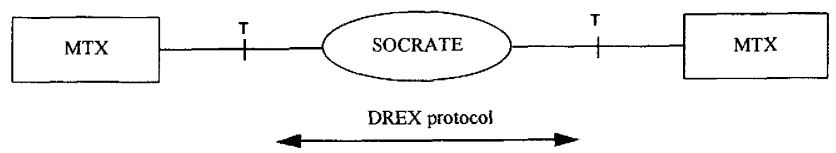

Figure 1 Reference configuration of DREX

The DREX protocol runs on a network called SOCRATE and connects several MTX (telecommunication means of military bases) at the T interface (see Figure 1). The DREX protocol contains a functional description of the offered services and the specification of the 
user interface for the call command. The SDL specification models the behaviour of the DREX protocol on the entire network SOCRATE.

Only a subset of the military supplementary services offered by the DREX protocol have been specified in SDL. These services are Priority, Roving User, Call Forwarding, Implicit Partioning of Users, Safety Path and User to User Signaling. A generic SDL specification has been written and instantiaced for each service. So, there is as many SDL specifications as services. This means that tests have been produced for each service separately and the interactions between services have not been studied. The SDL specification models only one connection between two MTX. They are called DR for the calling MTX and DE for the called MTX. The size of the SDL specification corresponding to each service is about 2000 lines.

\subsection{Test architecture and test purposes}

The test method chosen is called transversal method. The implementation of the protocol inside the network is seen as a black box. The tester models the behaviour of two MTX (seen as users from the network point of view), DR and DE connected to the SOCRATE network by two PCOs (Points of Control and Observation) at interface $T$. The communication between the MTX and the network is supposed to be asynchronous. The test configuration is shown in Figure 2.

The test purposes used as an input of our experiments are written in natural language These test purposes described the context of the test and an abstraction of the intended behaviour. The context of the test is concerned with properties of the subscription record of the users or properties of the network. An example of context is "The called MTX does not have access to this service " or "The network is saturated». The remainder of the test purpose describes sequencing properties between sendings and receptions of the tester and network. as well as properties on parameter of messages. For example, "The called MTX receives a SETUP message containing the parameter User to User Information (IUU). It sends an ALERT message with parameter IUU, Verify that the network sends to the calling MTX an ALERT message without parameter IUU *.

These test purposes have been used by the automatic tools for the test cases selection.

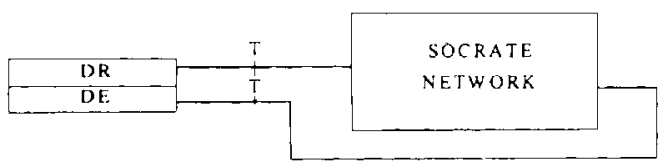

Figure 2 Test configuration for DREX

\section{PROTOTYPES DESCRIPTION}

\subsection{TGV}

\section{Overview}

TGV is a prototype tool for the generation of test suites developed by Inria. It is based on techniques that have proved useful in verification such as on-the-fly verification, product of transition systems and behavioural equivalence (see (Fernandez, 1996 and 1996b) for more 
details). It takes as input the state graph produced by GEODE of an SDL specification constrained by the test context and an automaton formalizing the behavioural part of a test purpose. The transitions of the test purpose automaton are labelled with some actions of the tester corresponding to visible actions of the IUT.

The TGV package outputs test suites given in TTCN standard language, distinguishing the control (behaviour) and data (constraints) parts. As usual, the intermediate form TTCNMP can be graphically displayed using TTCN-GR.

TGV has been developed in the toolbox CADP (Caesar Aldebaran Distributed Package). This was particularly useful because CADP is an open environment which does not depend on a particular language. Another advantage of CADP is the availability of libraries for the management of graphs and memory.

The external view of the TGV package is illustrated in Figure 3.

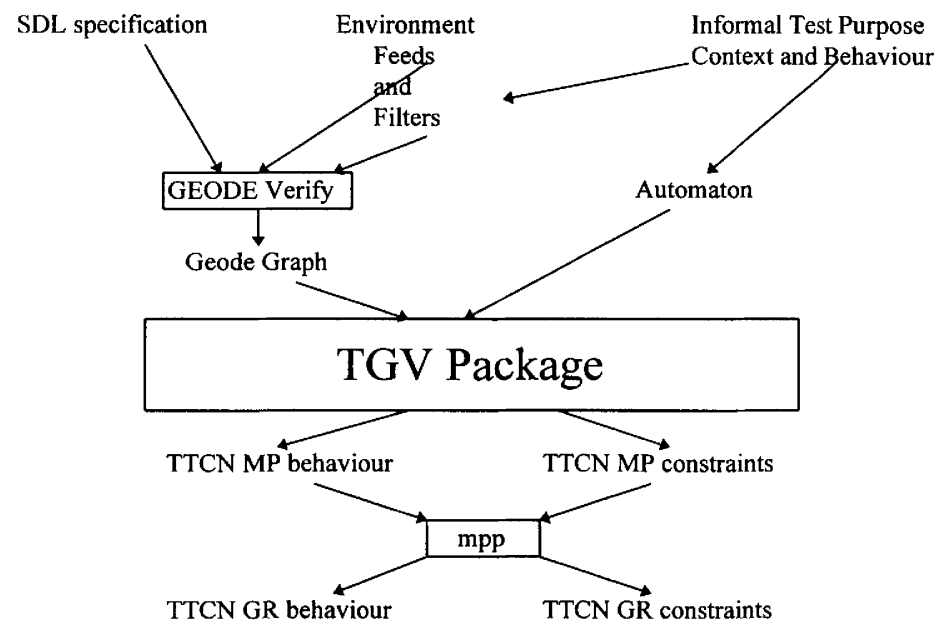

\section{Figure 3 External view of TGV}

\section{SDL model}

The SDL model is the same as the one used by the other tools. Its state graph is first generated by GEODE using exhaustive simulation. The exhaustive simulation also uses as inputs feeds and filters. Feeds are used to close the SDL specification with a restricted set of messages sent by the environment in order to limit the size of the state graph generated by GEODE. Filters specify some transitions that are considered as not firable. These filters are used to select the behaviours of the protocol within a context specified in the test purpose (see below).

The simulation of the model also assumes the reasonable environment hypothesis which says that, each time the environment sends a message to the network, it waits until stabilization. This means that no new message can be sent by the environment until it receives all specified sendings of the protocol. 


\section{Test purposes}

A test purpose defines a property on some observable interactions of the IUT. The constraint part gives some property on the state of the implementation. It is modelled by a set of filters.

The behavioural part which describes an ordering between interactions is modelled by an automaton using the Aldebaran format. This automaton is a direct acyclic graph with a set of distinguished accepting states with no successor.

Test generation process

The generation of a test case can be decomposed in several functional parts which are performed by different tools, as shown in Figure 4.

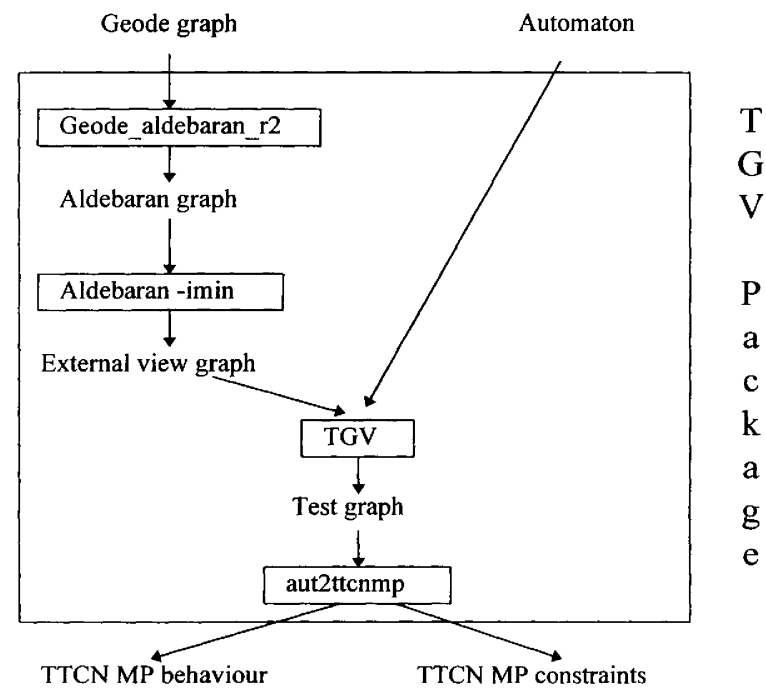

Figure 4 Test suite generation with TGV

Step 1: State graph generation with GEODE. The inputs to the GEODE exhaustive simulator are the SDL specification, feeds and filters. The output is a graph in GEODE format representing the whole set of behaviours of the specification in the context given by feeds and filters.

Step 2: Translation and mirror image. In this step, the graph generated by GEODE is translated into a graph in the Aldebaran format which represents the external view of the behaviour of the specification in the test environment. This is done to take into account the fact that the tester cannot interact direcly with the implementation but with a test environment in which the implementation is placed. But the specification models the internal view of the system, i.e. the behaviour of the system with its internal actions, without considering the way it interacts with the environment. Thus we have to put the specification in the same environment as it should be taken into account in the test generation. For our experiment, the communication between the tester and the implementation is asynchronous. This may introduce concurrency in the behaviour as we 
consider two interaction points. This has been modelled in the translation.In the following, we will consider the environment point of view: outputs are controlable actions initiated by the environment (which may be the tester) and sent to the IUT whereas inputs are observable actions, initiated by the IUT and received by the environment. Internal actions of the specification are replaced by invisible transitions denoted by $\tau$. Only interactions with the environment are to be captured. As we now consider the tester view, sending are to be replaced by receptions and vice versa.

Step 3: Minimization with Aldebaran. In this step, the graph produced at the preceeding step is minimized, using the Aldebaran tool, with respect to the $\tau *$ a equivalence. The minimization roughly means that only observable actions are preserved and states with same observable behaviours are collapsed. Collapsing is in fact not necessary, the important thing here is only the hiding of $\tau$ actions. This is crucial as we intend to make on the fly generation of test cases and on the fly minimization is not efficient. After this step, the minimized graph produced represents the external view of the protocol behaviour. We call it the external view graph.

Step 4: Test graph generation. This is the kernel of the tool. The algorithm is based on a depth-first traversal of a kind of synchronous product between the external view graph and the automaton representing the test purpose. In this product, states are composed of a state of the graph and a state of the automaton. A transition $t$ is firable in $\left(s_{g} ; s_{a}\right)$ and leads to $\left(s_{g}{ }^{\prime} ; s_{a}{ }^{\prime}\right)$ in two cases. Either $t$ is firable in the graph in state $s_{g}$ and leads to $s_{g}$ ' and $t$ is firable in the automaton in state $s_{a}$ and leads to $s_{a}{ }^{\prime}$, or $t$ is firable in $s_{g}$ and leads to $s_{g}{ }^{\prime}$ but $t$ is not firable in $s_{a}$, in which case $s_{a}{ }^{\prime}=s_{a}$.

During the traversal, two different things are done:

- the algorithm checks that at least one sequence allows to reach a state in which the automaton component is an accepting state. In case of success, this ensures that the test purpose is at least realizable. We could impose this for all sequences but this one was taken because in some cases the test context could not be modelled by filters. Thus some sequence did not satisfy the test purpose.

- meanwhile, a skeleton graph is synthesized. This graph contains sequences of the synchronized product that contain an accepting state of the automaton. In this graph, there is never the choice between two outputs of the tester because the tester controls outputs.

Some transitions are decorated with verdicts with the following informal meaning. (PASS): the test purpose is satisfied by the current sequence. But a sequence leading to the initial state (Postamble) must be applied in order to carry on another test case. It is a temporary verdict as the application of the postamble may produce FAIL verdicts. PASS: a definitive verdict meaning that the initial state has been reached after a (PASS) verdict. The sequence between (PASS) and PASS is a Postamble. FAIL: means non-conformance of the IUT on inputs of the tester. For the conformance relation chosen this means that after a sequence of interactions valid in the specification, this input does not corresponds to a valid output of the specification. INCONCLUSIVE: verdict produced when an input of the tester is allowed in the specification but cannot lead to a (PASS) or leads to a behaviour that is not considered in the test case because testing cannot be exhaustive in practice.

After that, the graph is decorated with timers. Timers are used in test cases when a reaction of the implementation is expected but one does not want to wait for an unbounded time because an error that is not observable may have occurred. The difficulty in the management of timers is that concurrency and non-determinism should not be treated in the same way. Timers are managed by TGV in the following way. A timer tm is associated to each possible input of a message $\mathbf{m}$. The timer tm is started in the last transition which necessarily precedes the reception of $\mathbf{m}$. In a state where the input of $\mathbf{m}$ is possible, a 
timeout of tm is also possible. In each transition sequence, a timer tm is cancelled in a transition following its start and as soon as the reception of $\mathbf{m}$ is no more possible in the future. This is were concurrency and choice must be distinguished.

Step 5: TTCN generation. In this last step, the tool aut2ttcnmp takes as input the test graph generated at the preceding step, and produces two files : one describing the behaviour part of the test case (obtained by unfolding the test graph in a tree), the other describing the constraints part. Both files are in TTCN MP format and are translated into files in TTCN GR format by mpp, a tool from VERILOG.

\subsection{TOPIC V2}

\section{Overview}

Developments of TOPIC V2 have started in the RACE II/TOPIC project, based on the results of the RACE II/PROVE project. A product version of this prototype called TTCgeN is now included in the VERILOG ObjectGEODE product line.

TTCgeN generates abstract tests in TTCN-IS MP from (i) the SDL model describing the protocol to be tested and (ii) test objectives (or purposes) formalized with MSCs.

The TTCgeN test generation algorithm is based on the exhaustive exploration of SDL model behaviour. This exploration is made by going through a subset of the overall state graph. This subset is obtained by constraining the complete protocol model behavior with the help of the test purposes. TTCgeN is thus based on automatic exhaustive simulation techniques.

\section{SDL model}

The SDL model describes the protocol to be tested, not its environment. This model is open, which means that it exchanges signals with the environment via dedicated channels.

In order to lower the number of possibilities regarding possible data conveyed by signals incoming from the environment, test purposes include data samples able to be received by the protocol model.

Like for TGV, the reasonable environment hypothesis has been put on the environment. Of course the delay between two emissions from the environment must be very short compared to timeouts involved within the protocol model.

No other restrictions are imposed on the SDL model, except that a finite state graph (constrained by test purposes) can be derived from this SDL model.

\section{Test purposes}

A test purpose must be described with the help of an MSC. Figure 5 shows an example of an MSC describing a test purpose. Signal sequence $a$ and $b$ is a preamble to set the IUT in the desired testable state. The next sequence shows that after having received signal $\mathrm{x}$, the IUT should send back signal y carrying data value 15 .

This test purpose MSC can be described by the user through two different ways by:

1. using the ObjectGEODE MSC Editor directly or,

2. generating this MSC from within the ObjectGEODE SDL Simulator after a simulation session corresponding to the desired signal sequence. This particular way guaranties that the test purposes are correct vs. the SDL model.

\section{Test generation process}

On a conceptual point of view, the generation of a test consists of the following operations : to find in the overall state graph all the event sequences corresponding to the test purpose (PASS verdict); and to complement these sequences with all valid but non expected events (INCONCLUSIVE verdict), and non valid events (FAIL verdict). 


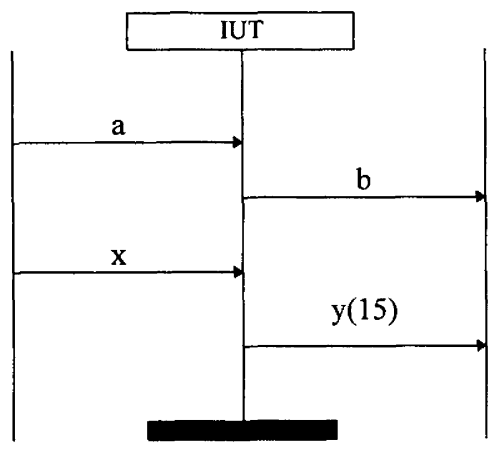

Figure 5 Example of an MSC describing a test purpose

The overall test generation process is described in Figure 6.

One of the major difficulties is to find these sequences as most efficiently as possible, that is by exploring as less states as possible. This can be achieved doing so-called cosimulation between the SDL protocol model and a test purpose previously translated as an observer. An observer is described in a language called Geode Observation Automata Language (GOAL) (Algayres, 1995), derived from studies conducted at CNET. In the test generation process, GOAL observers are automatically derived from MSCs.

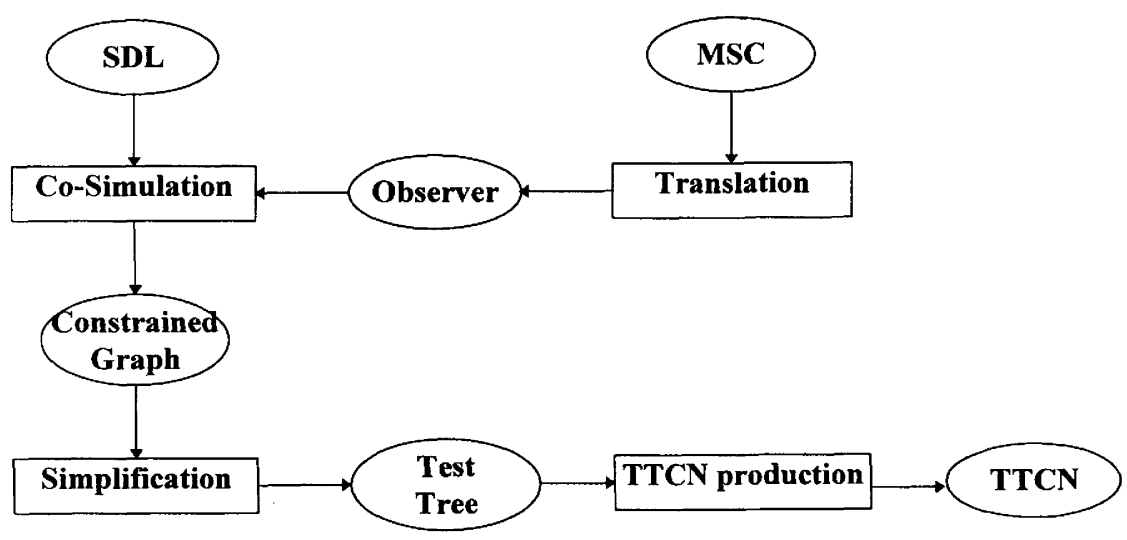

Figure 6 Overall test generation process with TOPIC V2 (TTCgeN)

Co-simulation allows the exploration of only a subset of the overall state graph, thus enabling the use of this technique on infinite graphs, i.e. on SDL models that cannot be 
exhaustively simulated. The subset is built based on exploration constrained by the GOAL observers.

The constrained graph resulting from co-simulation contains sequences (i) leading to the PASS verdict and the preamble, (ii) valid but not expected answers from the IUT (INCONCLUSIVE verdict), and (iii) valid but not expected stimuli from the Tester.

Simplification is then applied to the resulting constrained graph for removing the later sequence case.

Lastly, TTCN production is in charge of (i) translating constraints (signal parameters) in TTCN, (ii) generating PDU declaration, (iii) complementing the test sequence with the FAIL verdict in case of no response from IUT (use of timers) or wrong response from IUT (?OTHERWISE).

\subsection{TVEDA V3}

\section{Overview}

TVEDA V3 is a test generation tool which has been developped by CNET (France Telecom) since 1989. TVEDA produces TTCN test suites from Estelle or SDL formal specifications. TVEDA incorporates two distinct test generation techniques, which have been studied successively:

1. the "syntactic" technique produces test cases by a simple syntactic transformation of the specification. In this technique, emphasis is put on heuristics which allow an automatic computation of the test purposes (see section Test purposes). On the contrary, no semantic computation (e.g. simulation) is done on the specification. Therefore the test cases which are produced are incomplete, and must be manually completed. This technique has been described in (Phalippou, 1990).

2. the technique "by simulation" has been developped more recently. In addition to the automatic computation of the test purposes (same heuristics as in the syntactic technique), this new method uses advanced exhaustive simulation functions in order to compute complete compilable TTCN test suites. The simulation functions are not part of the TVEDA tool, but are provided by coupling TVEDA with available verification tools (such as GEODE, from VERILOG company, for SDL language (Algayres, 1993). This technique has been described in (Clatin, 1995).

In this project only the syntactic method has been used, because the technique by simulation was not available when the project started (end of 1994).

The syntactic technique of TVEDA is currently being industrialized by an european consortium, financed by the European Commission, named CATG, whose prime contractor is SEMA GROUP. The resulting industrial tool will be called TTCN Maker, and will be publicly available by the end of 1996 .

\section{$S D L$ model}

TVEDA takes as input a formal specification written in SDL. The syntactic technique puts some constraints on the SDL constructs which can be handled by the tool. These constraints are of two kinds:

1. restrictions on SDL constructs. The tool ignores several constructs, such as abstract data types, dynamic process management, etc.

2. restrictions on SDL style. The syntactic technique generates test cases for a single SDL process. Several processes can be accepted, but TVEDA will produce distinct test cases for each one of the processes, and then merge the test cases into a single test suite. This means that the tool cannot compute the combined behaviour of the various processes. In addition, to be relevant for the syntactic method, each transition of the specification must 
start with an input from a given stable state, produce eventually outputs, and end into a stable state. This means that spontaneous transitions as allowed by SDL 92 are not allowed by TVEDA. Complex procedures (i.e. containing inputs or outputs) are not allowed either.

\section{Test purposes}

One of the main features of TVEDA, which is not provided by the other tools described in this paper, is the automatic computation of the test purposes. For doing this, the tool incorporates some heuristics, based on the syntactic structure of the SDL specification. Basically, one test case is produced in order to check each branch of the SDL (the resulting coverage is thus close to the "branch coverage" often used for the test of sequential programs). This basic test selection mechanism can be modified by the user of TVEDA in the following ways:

1. restrict more or less the part of the specification which is tested: choice of states, input signals, procedures, etc.

2. enumerate more or less data values which appear in the SDL branches: test several values of parameters on input signals, or on the contrary merge test cases corresponding to several branches if the observable behaviour is the same in these branches, etc.

These heuristics have been defined after observation of the stategy used by human experts in order to define the test cases. They have been validated by applying TVEDA to several real protocols and then by comparing TVEDA test purposes with the test purposes of standardized manually generated test suites for the same protocols. For instance, in the case of ISDN D protocol (level 2 user side, level 3 user and network side), more than $90 \%$ of the manual test purposes have been correctly generated by TVEDA.

The test purposes produced by TVEDA look like : "Check that the IUT sends a $<$ message and significant parameter values $>$ in response to a $<$ message and significant parameter values $>$ in state $<$ state $>$. The IUT is supposed to enter state $<$ state $>$ ".

\section{Test generation process}

Once the test purposes have been selected, TVEDA computes the behaviour of the test cases. This is done in several steps.

Step 1: Analysis of the SDL specification, and computation of an abstract extended finite state machine (EFSM). During this step, the test architecture is taken into account (which signals can be controlled, which signals can be observed: this is the definition of the PCOs). The level of abstraction at which the specification is tested can be influenced, through the decision to "control" or not variables or signals: this mechanism, which has been adjusted in an empirical way, is very important, and has a significant influence on the output of the tool. It is a characteristic of TVEDA, hardly studied in classical test generation methods. More information can be found in (Phalippou, 1990).

Step 2: Production of test cases in an internal abstract format. Incomplete test cases are produced, which have the following structure :

- call to a preamble which brings the IUT into the start state of the tested transition. This preamble is not completely computed with the "syntactic" technique, since this would require computation of the SDL behaviour (on the contary it is produced by the technique "by simulation").

- send and receive events corresponding to the tested transitions. At this level, non determinism is taken into account. Non determinism can result from uncontrolled variables (with the consequence of decisions in which it is not possible to know which branch will be executed) or from uncontrolled events (which have visible consequences at the PCOs). Constraints are also produced in an incomplete way, using heuristics 
where possible and informal comments in the remaining cases.

- call to a check sequence which is supposed to check the final state of the tested transition and bring back the IUT in its initial state. Here again this check sequence is not completely produced by the "syntactic" technique (it is produced by the technique "by simulation").

Step 3: Formatting of TTCN suite. Elements which are purely linked to TTCN are added to the internal abstract test suite. This includes translation of the constraints into ASN.1 format, computation of the test suite structure, of the test case names, production of the different TTCN parts (overview, declarations, constraints, test cases, test steps), and finally production of hypertext links which relate each test case with the corresponding tested part of the formal specification.

The output of TVEDA is a test suite in standard TTCN-IS MP format.

\section{EXPERIMENTS RESULTS}

\subsection{Contribution made by the different prototypes}

The experimental work carried out in this study has shown that the three prototype test generators worked as expected on a real protocol. The test generation times are short and the memory requirements are low. The main advantages of each prototype with regard to the others are reviewed below:

Advantages of TOPIC V2

- graphical test purpose (MSCs),

- generation of declarations.

\section{Advantages of TGV}

- generation algorithms based on the technology of on-the-fly verification,

- generation of tests taking into account asynchronism between IUT and tester,

- high-level control of timers.

\section{Advantages of TVEDA V3}

- test purposes computed automatically (this has allowed 2 new test cases to be discovered in the context of the experimental work),

- generation of declarations,

- generation of a full suite (and not of isolated tests),

- takes into account the level of abstraction at which the specification has to be viewed from the point of view of the test.

\subsection{Quantitative results}

Figure 7 represents a comparison of the effort required for each phase and for each test generation method. At first sight, this diagram seems to show that automatic generation is of little value. The following comments demonstrate that this is not the case:

- more than half of the workload is given over to writing the SDL model : as well as enabling automatic generation of tests, this model allows the quality of the specification of the protocol to be improved by means of syntactic and semantic verification and by simulation carried out using a tool such as GEODE; the effort needed to adapt the specification to the TVEDA format would not have been necessary if the specification had been written directly for TVEDA, 
- about a third of the workload is given over to the finishing work on the test suite in order for it to be accepted by a TTCN compiler (addition of declarations, postambles, semantic verification, etc...) : with a 'full' tool rather than the prototypes used in this study, hardly any of this finishing work would be required,

- this comparison relates to 54 tests on the DREX protocol : if we extrapolate the results, the value of the automatic test generators becomes more obvious. This is described in the 'Perspectives' section.

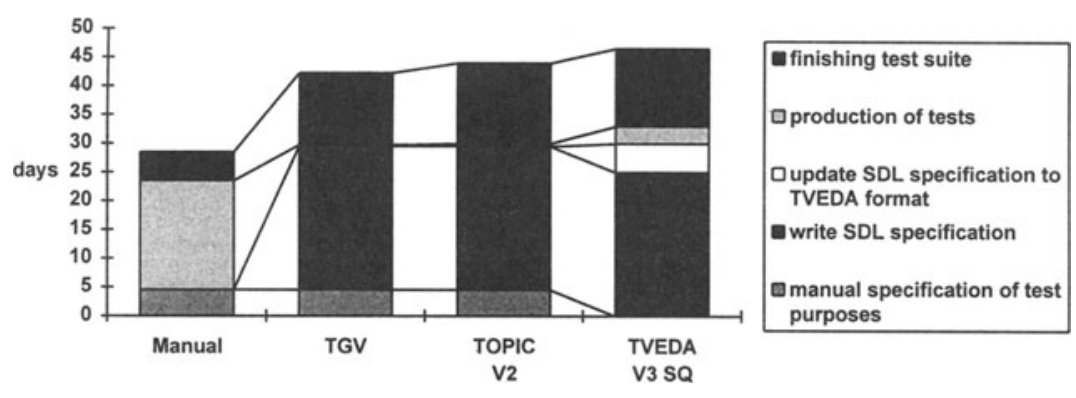

Figure 7 Effort required to obtain an abstract test suite

Also, the figures shown allow for the fact that the people carrying out the experiments were specialists in their tools, but they were not necessarily specialists in all the domains involved in this study, namely the SDL language, the DREX protocol, and testing. In spite of this, tests which are totally comparable with manual tests have been generated.

\subsection{Qualitative results}

The comparison of the test suites automatically generated with hand-written test suites has enabled some errors to be detected in the manual tests, which shows the value of the automatic methods for producing correct tests.

This comparison has also enabled the prototypes to be refined as the tests were conducted by incorporating rules into the algorithms which had not originally been thought of.

The use of automatic test generation methods offers significant advantages:

- an SDL model of the protocol is obtained which can be simulated and verified by powerful tools available on the market such as ObjectGEODE; this allows us to ensure that the specification of the protocol is correct, which is difficult without a formal model,

- when refining the protocol, it is less dangerous and less costly to update the SDL model (which can be simulated to ensure that it is still working) and then to rerun the generator, than it is to modify a TTCN test suite by hand,

- an automatic generator makes the tests safer by avoiding the human error inevitable in a manual approach and this saves a great deal of time, even if the figures cannot show this ; it also enables more tests to be generated, which gives better coverage of the protocol (the coverage seems to be correct for the DREX CS, but for other protocol layers it may be envisaged that achieving adequate 'manual' coverage would be difficult). 


\section{PERSPECTIVES}

Not everything has yet been resolved in the prototypes, but this experiment and the comparison between the prototypes have brought to light their advantages and limitations and shown how to design a tool incorporating the best ideas from TOPIC V2, TGV and TVEDA V3.

Two steps towards the industrialization of such a tool have been identified.

\section{First step: A 'full' tool}

The 'full' tool based on TOPIC V2 integrates:

- generation algorithms based on the technology of on-the-fly verification of TGV and TOPIC V2,

- the automatic computation of declarations and postambles,

- the control of the abstraction level and the full suite building proposed by TVEDA V3,

- the taking into account of asynchronism between IUT and tester, and the high-level control of timers proposed by TGV.

This 'full' tool requires the specification of test purposes with the help of MSCs.

\section{Second step: An 'ideal' tool}

The 'ideal' tool integrates the features of the 'full' tool and:

- the automatic computation of test purposes with an optimization of the method proposed by TVEDA V3,

- the editing of generation parameters via a user interface,

- the interactive piloting of the test generation by the user to allow the incremental building of the test suite.

Figure 8 shows an extrapolation of the production effort for 895 tests on the DREX protocol, for each phase and for test generation with the manual method, with the 'full' tool and with the 'ideal' tool.

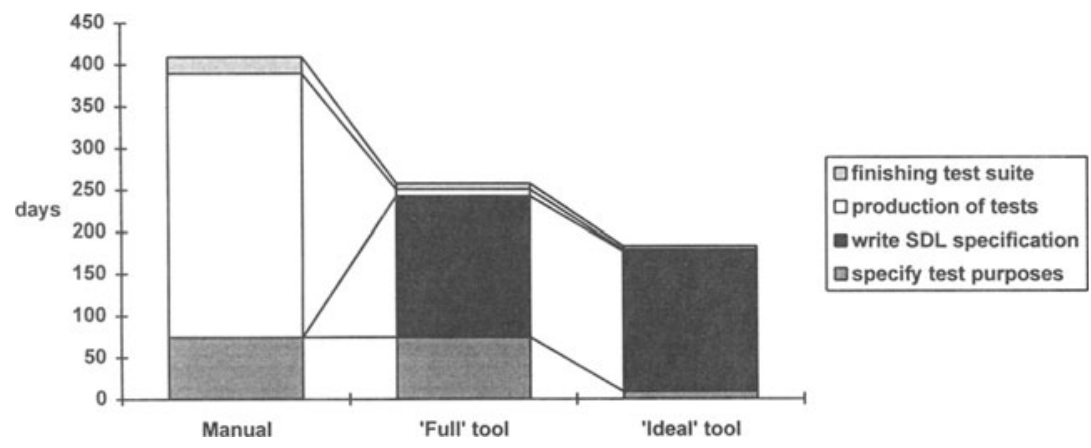

Figure 8 Effort for 895 tests, with a 'full' and an 'ideal' tool

For manual test generation a rule of three has been used except for the effort of the finishing work on the test suite, which has been estimated at 20 days. 
With the 'full' tool the workload of writing and debugging the SDL model is estimated at 168 days. A rule of three has been used to calculate the specification time for test purposes and the implementation time for the generation of tests (TOPIC V2 figures). The effort of the finishing work on the test suite is estimated at 7 days.

With the 'ideal' tool the workload for writing and debugging the SDL model is the same as for the 'full' tool. The effort of configuring the tool to generate the test purposes is estimated at 9 days. The implementation time for the generation of tests and the finishing work on the suite is estimated at 6 days; in fact this enables the tests to be regenerated if a problem has appeared.

Regarding productivity, the current generation tools (described in this paper) already provides a productivity improvement of $+25 \%$ over conventional (manual) test writing techniques, while the 'full' tool should provide $+38 \%$ productivity gain and the 'ideal' tool $+55 \%$.

\section{ACKNOWLEGMENTS}

We wish to thank the DGA/STSIE for financing this project, particularly Cpt. Plessix, Mrs. Courtier and Mr. Caporini who launched the project and were responsible for it, as well as the other DGA participants : Mr. Le Pape and Mr. Ropars from DGA/CELAR.

We wish also to thank the other partners in the experiment related here. In particular, C. Jard and G. Viho from IRISA/Pampa, J. Sifakis from Vérimag and P. Barbier from CAP SESA.

\section{REFERENCES}

CCITT/SGx/WP3-1 (1988) Specification and Description Language, SDL, in CCITT Recommendation Z.100.

Clatin, M., Groz, R., Phalippou, M. and Thummel, R. (1995) Two approaches linking a test generation tool with verification techniques, in Proceedings of IWPTS '95, Evry.

Fernandez, J.C., Jard, C., Jéron, T. and Viho, G. (1996) Using on-the-fly verification techniques for the generation of conformance test suites, to appear in Conference on Computer-Aided Verification (CAV '96), New Brunswick, New Jersey, USA.

Fernandez, J.C., Jard, C., Jéron, T. and Viho, G. (1996b) An experiment in automatic generation of test suites for protocols with verification technology, to appear in Science of Computer Programming, Special Issue on Industrial Relevant Applications of Formal Analysis Techniques (ed. J.F. Groote and M. Rem), Elsevier Science publisher.

Algayres, B., Lejeune, Y., Hugonnet, F. and Hantz, F. (1993), The AVALON Project: A VALidatiON environment for SDL/MSC descriptions, in Proceedings of 6th SDL Forum, Darmstadt.

Algayres, B., Lejeune, Y., Hugonnet, F. and Hantz, F. (1995), GOAL: Observing SDL behaviour with GEODE, in Proceedings of 7th SDL Forum, Oslo.

Montiel, J., Roth, R. and Donaldson, A.J.M (1994). Methods for QoS Verification and Protocol Conformance Testing in IBC - Results and Further Recommendations. Race Project R2088 TOPIC, Deliverable 15, DocR2088/DAT/TMS/DS/P/015/b1.

Phalippou, M. and Groz, R. (1990) Evaluation of an empirical approach for computer-aided test cases generation, in Proceedings of IWPTS' 90 , Washington.

Phalippou, M. (1994) Test sequence generation using Estelle or SDL structure information, in Proceedings of FORTE'94, Berne. 